\title{
FATORES DETERMINANTES DA PROPENSÃO AO CONSUMO DE CIRURGIAS PLÁSTICAS ESTÉTICAS
}

\section{DETERMINANTS OF PROPENSITY TO SEEK AESTHETIC PLASTIC SURGERY}

\section{Mateus Canniatti Ponchio}

Doutor em Administração pela Escola de Administração de Empresas de São Paulo da Fundação Getulio Vargas - FGV-EAESP

Professor da Escola Superior de Propaganda e Marketing de São Paulo - ESPM/SP

E-mail: mponchio@espm.br (Brasil)

\section{Carla Giglioli Martins}

Graduada em Administração pela Escola Superior de Propaganda e Marketing de São Paulo ESPM/SP

E-mail: carlagm89@gmail.com (Brasil)

\section{Cecilia Bandeira de Melo Vieira}

Graduada em Administração pela Escola Superior de Propaganda e Marketing de São Paulo ESPM/SP

E-mail: ceciliabandeirademelo@ hotmail.com (Brasil)

\section{Daniela Menezes}

Graduada em Administração pela Escola Superior de Propaganda e Marketing de São Paulo ESPM/SP

E-mail: daniela.menezes @live.com (Brasil) 


\title{
FATORES DETERMINANTES DA PROPENSÃO AO CONSUMO DE CIRURGIAS PLÁSTICAS ESTÉTICAS
}

\begin{abstract}
RESUMO
O presente estudo analisa o consumo de cirurgias plásticas com finalidade estética entre as consumidoras de São Paulo e Recife, duas grandes cidades brasileiras que possuem características econômicas, demográficas e culturais distintas. Nesse contexto, os objetivos são compreender a influência do materialismo, da autoestima e de variáveis de controle sobre a decisão de realizar uma cirurgia plástica estética. Um questionário foi elaborado com perguntas fechadas e aplicado pela Internet, resultando em dados disponíveis para análise de 347 respondentes. Com 173 moradoras de São Paulo e 174 de Recife, constatou-se que a cidade da respondente não ajuda a discriminar a probabilidade de que efetue uma cirurgia plástica com finalidade estética; por outro lado, a dimensão 'comparação social' da escala de autoestima, bem como o nível de materialismo, provaram-se variáveis preditoras significantes desse tipo de consumo. Por fim, são apresentadas implicações gerenciais e acadêmicas dos achados desse estudo, bem como possibilidades para novas investigações.
\end{abstract}

Palav ras-chave: Cirurgia Plástica Estética. Autoestima. Materialismo. Comportamento do Consumidor. Marketing.

\section{DETERMINANTS OF PROPENSITY TO SEEK AESTHETIC PLASTIC SURGERY}

\section{ABSTRACT}

The present study analyses the consumption of aesthetic surgeries among females from the cities of São Paulo and Recife, two large Brazilian cities that possess different economic, demographic, and cultural characteristics. In such context, the objectives of this paper are to comprehend the influence of materialism, self esteem, and of control variables on the decision to undertake an aesthetic surgery procedure. A questionnaire was made available online and was completed by 347 respondents, 173 from São Paulo and 174 from Recife. The data analyses show that the city of the respondent does not help to predict the probability of engaging in an aesthetic surgery; on the other hand, the 'social comparison' dimension of the self esteem scale, as well as materialism, do contribute to predict the probability of occurrence of such event. The academic and managerial implications of the findings are discussed, as well as possibilities for future investigations.

Key-words: Aesthetic surgery. Self esteem. Materialism. Consumer behavior. Marketing.

REMark - Revista Brasileira de Marketing, São Paulo, v. 12, n. 4, p. 44-63, out/dez. 2013. 


\section{INTRODUÇÃO}

De acordo com o instituto Euromonitor (2010), estima-se que o Brasil represente $10 \%$ do consumo mundial de produtos de beleza e cuidados pessoais, cerca de US\$ 38 bilhões. A mulher brasileira parece não hesitar em utilizar artifícios disponíveis no mercado para poder se sentir melhor com sua aparência (Machado \& Pereira, 2010). Segundo Swami, Chamorro-Premuzic, Bridges, \& Furnham (2009), podem-se observar evidências de que quanto pior a autoestima de um indivíduo, maior a probabilidade de que a percepção de sua imagem corporal seja ruim, e consequentemente, maiores as chances de que busque por meio de cosméticos e cirurgias plásticas estéticas (CPE) o aumento da autoestima.

O mercado de estética passa por transformações para conseguir acompanhar desejos das mulheres como pelo uso de tratamentos estéticos e de produtos de beleza. Esses, apesar dos custos financeiros, são vistos como boa forma de trazer benefícios emocionais, sociais e materiais (Teixeira, 2001).

As mulheres são a grande maioria adepta às cirurgias estéticas; nos Estados Unidos, eram responsáveis por cerca de um milhão de procedimentos em 2011, 92\% do total, e por cerca de 740 mil procedimentos no Brasil, ou $82 \%$ do total (Brasil..., 2013). Nos Estados Unidos, houve crescimento de 69\% no número desses procedimentos do ano de 2000 para 2009 (ASAPS American Society of Aesthetic Plastic Surgery, 2010). O Brasil destaca-se nesse cenário por ser o país que possui o maior número de procedimentos per capita em todo o mundo, perdendo apenas para Estados Unidos e China na quantidade de cirurgias plásticas estéticas realizadas por ano (Brasil..., 2013).

$\mathrm{Na}$ literatura acadêmica, vários fatores são apontados como possíveis causas para o aumento na popularidade da cirurgia estética, tais como a autoestima e o materialismo (Delinski, 2005; Swami et al., 2009). Na última década, observa-se aumento na cobertura da cirurgia estética pela mídia (Crockett, Pruzinsky, \& Persing, 2007). Segundo Sarwer et al. (2003), os indivíduos estão cada vez mais expostos, por meio de suas redes sociais, à possibilidade de fazer a cirurgia estética. As propagandas e revistas que prometem ajudar a aumentar a atratividade, apresentando os mais recentes procedimentos cirúrgicos, e os reality shows da televisão, que comemoraram a transformação da aparência física, são vistos, hoje, com naturalidade.

A academia tem dedicado esforço para compreender diferenças em fatores demográficos, psicossociais, e individuais associados à realização de cirurgia plástica estética. Por exemplo, quanto aos aspectos demográficos, além da inclinação maior das mulheres por cirurgias de foro estético (Brown, Furnham, Glanville, \& Swami, 2007; Swami et al., 2009), um estudo de Swami, 
Campana e Coles (2012) mostra que mulheres caucasianas aceitam melhor os procedimentos de cirurgia estética do que mulheres africanas e sul-asiáticas, mesmo controlando para variáveis tais como autoimagem do corpo e autoestima.

A aceitação de tais procedimentos cirúrgicos também está ligada ao materialismo. Os valores materialistas são expressos pela aspiração ao sucesso financeiro, alto status social e aparência (Ponchio \& Aranha, 2008). De acordo com Delinsky (2005), da mesma forma, certos adjetivos, como materialista, autoconsciente e perfeccionista, foram também eleitos para refletir um estereótipo generalizado sobre os pacientes da CPE. Sabe-se que a cirurgia plástica, como técnica de modificação, tem potencial para aumentar a atratividade física e permitir a seu usuário adquirir os benefícios sociais e financeiros a ela associados (Henderson-King \& Brooks, 2009). Logo, parece poder aferir-se que o materialismo faz aumentar a propensão pelo consumo de CPE.

A maior parte das pesquisas sobre aceitação à cirurgia estética foi conduzida com consumidores de mercados desenvolvidos. De acordo com Steenkamp (2005), as teorias estabelecidas e as generalizações empíricas decorrentes de dados extraídos naqueles mercados não são necessariamente aplicáveis ao contexto de mercados emergentes. Assim, pesquisas sobre comportamento do consumidor em mercados como o brasileiro são relevantes (Burgess \& Steenkamp, 2006).

Nesse contexto, o presente estudo aborda o consumo de cirurgias estéticas entre consumidoras de São Paulo e Recife, duas grandes cidades brasileiras que possuem características econômicas, demográficas e culturais distintas. O objetivo é compreender fatores determinantes do consumo de cirurgias plásticas estéticas.

\section{REVISÃO DA LITERATURA}

São revisados, nas seções seguintes, os conceitos de cultura de consumo, autoestima e materialis mo.

\subsection{CULTURA DE CONSUMO E AUTOESTIMA}

No anos 1980, Arnould e Thompson desenvolveram a Teoria da Cultura do Consumidor (CCT - Consumer Culture Theory), a qual busca entender a relação entre as ações do consumidor, o mercado e os significados da cultura. Em estudo recente, os autores conceituam cultura de consumo como "um sistema comercial interconectado de imagens, textos e objetos consumidos pelos grupos para criar um senso coletivo de seus ambientes e para orientar as experiências e a vida de seus

REMark - Revista Brasileira de Marketing, São Paulo, v. 12, n. 4, p. 44-63, out/dez. 2013. 
membros" (Arnould \& Thompson, 2005, p. 869, tradução nossa). Em outras palavras, para os autores, cultura de consumo significa um arranjo social no qual as relações entre a cultura vivente, os recursos sociais, os significados dos estilos de vida, seu simbolismo e recursos materiais, dos quais os indivíduos dependem, são mediados pelo mercado (Wattanasuwan, 2005).

Às atividades, às experiências e aos objetos participantes do sistema citado acima são atribuídos sentido e significado, o que torna possível identificar e classificar indivíduos, grupos e culturas (Hirschman \& Holbrook, 1981; Holbrook \& Hirschman, 1982). Da mesma forma, os significados atribuídos aos produtos e às experiências podem ser vistos como a base material em que se constrói a cultura (McCraken, 2003). A cultura diz respeito a valores que surgem do modo de vida de um povo, que dão a este uma moldura de identidade e que o norteia em relação ao que é bom ou mal, real ou falso (Slater, 2002). Os valores dominantes de uma sociedade são resultados das práticas de consumo, portanto o consumo tem importante papel na construção do autoconceito dos indivíduos, uma vez que, eles são representados por suas atitudes de compra (Birdwell, 1968).

De acordo com Solomon (2008), autoestima é:

A positividade do autoconceito de uma pessoa. Pessoas com baixa autoestima acreditam que não terão bom desempenho e tentam evitar o constrangimento, o fracas so ou a rejeição. [...] Por outro lado pessoas com alta autoestima esperam o sucesso, correm mais riscos e têm mais vontade de ser o centro das atenções. A aceitação pelos outros costuma influenciar a autoestima. (Solo mon, 2008, p. 177).

Segundo Dini, Ferreira e Quaresma (2004, p. 48) autoestima pode ser definida como “o sentimento, o apreço e a consideração que uma pessoa sente por si própria, ou seja, o quanto ela gosta de si, como ela se vê e o que pensa sobre ela mesma”. Para Xue, Zhou e Zhou (2002, p. 247, tradução nossa), "autoestima é o resultado da comparação entre o Eu ideal e o Eu real. Quanto menor a distância entre o ideal e o real maior será a autoestima. Reciprocamente quanto maior a distância menor a autoestima". O eu ideal é a concepção da pessoa do modo como gostaria de ser; já o eu real refere-se à apreciação mais realista das qualidades que temos ou não. $\mathrm{O}$ eu ideal é parcialmente moldado por elementos da cultura do consumidor (Solomon, 2008).

Rosenberg e Pearlin (1978) encontraram que a influência da classe social sobre a autoestima é diferente entre crianças e adultos. Para atingir esse resultado foi necessário considerar quatro teorias para a formação da autoestima:

a) Processos de comparação social, avaliação de opiniões, habilidades e status. O indivíduo costuma comparar os elementos de sua vida em relação aos que vivem em sua volta.

b) Avaliações Refletidas, "teoria segundo a qual nos vemos como percebemos que os outros nos veem" (Rosenberg \& Pearlin, 1978, p. 63, tradução nossa), ou seja, há forte relação entre o "eu percebido" e a real imagem que o indivíduo tem de si mesmo.

REMark - Revista Brasileira de Marketing, São Paulo, v. 12, n. 4, p. 44-63, out/dez. 2013. 
c) Autopercepção, teoria segundo a qual "aprendemos sobre nós mesmos da mesma forma que os outros aprendem sobre nós - pela observação e interpretação de nosso comportamento e resultados" (Rosenberg \& Pearlin, 1978, p. 65, tradução nossa).

d) Centralidade Psicológica (Autoconceito). O autoconceito é uma estrutura complexa e multifacetada composta por disposições (inteligência, otimismo, moral, entre outros) e elementos de identidade social (raça, sexo, religião, idade, classe social, entre outros).

Essas teorias também podem ser complementadas pelo estudo de Rosenberg, Schooler, e Schoenbach (1989) segundo a qual os indivíduos se julgam baseados na comparação com outros e que os sentimentos dos indivíduos por si mesmos são fortemente influenciados pelo julgamento de outros.

A autoimagem e a busca por sua melhora é um importante fator influenciador da compra (Heath \& Scott, 1997; Mittal, 2006); o indivíduo busca atingir a autoimagem ideal por meio dos significados dos bens de consumo. Esta é a vertente de estudo apoiada por Belk (1983); esse autor destaca a "extensão do Eu", ou seja, a ampliação da identidade do indivíduo por meio de suas posses, lugares, pessoas e grupos. Segundo Belk (1988), posses podem ser definidas como as coisas que podemos chamar de nossas e controlar. As posses passam a fazer parte do autoconceito do indivíduo a partir do momento em que forem consumidas.

Um estudo feito por Delinsky (2005) apontou que quanto maior é a importância da aparência para a autoestima das mulheres, maior é a aprovação delas em relação à cirurgia plástica. Segundo o autor, foi constatado também que quanto maior a autoestima da mulher, maior é a sua aprovação para com a cirurgia plástica estética.

\subsection{MATERIALISMO}

O termo "materialismo" denota a importância que um indivíduo atribui às suas posses (Belk, 1984). Segundo Belk (1984), os níveis mais altos de materialismo são assumidos quando as posses ocupam lugar central na vida de uma pessoa e se tornam a sua maior fonte de satisfação ou insatisfação. Na visão de um materialista as posses são centrais em sua vida, e o aumento do consumo eleva a sua satisfação pessoal.

Para os materialistas, as posses materiais são utilizadas para caracterizar e comunicar seus valores (Richins, 2004). De acordo com Richins e Dawson (1992), o materialismo possui três dimensões: sucesso, centralidade e felicidade. Na dimensão do sucesso, os materialistas tendem a julgar a si próprios e aos outros pelo número e qualidade de seus bens acumulados; na de centralidade, os materialistas fazem das posses o foco central de suas vidas; e na dimensão de

\footnotetext{
REMark - Revista Brasileira de Marketing, São Paulo, v. 12, n. 4, p. 44-63, out/dez. 2013.
} 
felicidade, as relações dos materialistas com os bens são essenciais para sua satisfação e se u bemestar. Assim, o valor dos bens, para os materialistas, reside na capacidade que eles têm de conferir determinado status e de projetar a autoimagem por eles desejada.

Richins e Dawson (1992) sugerem que os consumidores mais materialistas podem obter prazer ao adquirirem ou mostrarem um bem, e não ao usá-lo. Os mais materialistas são mais autocentrados e preferem utilizar seus recursos para si próprios, ao invés de compartilhá-los com os outros, mesmo que sejam amigos íntimos ou familiares. Quando Fournier e Richins (1991) entrevistaram diferentes consumidores para descobrirem como eles enxergavam os consumidores materialistas, os entrevistados os caracterizaram como aqueles que querem possuir o que há de melhor, os bens mais caros e outras posses que confiram status. Os mais materialistas, em comparação com os menos materialistas, se veem como mais gastadores e favoráveis à aquisição de empréstimos em diversas situações, como para comprar artigos de decoração, carros, barcos, uma segunda casa ou, até mesmo, para viajar de férias. Deste modo, embora os mais materialistas não se diferenciem substancialmente dos menos materialistas nos seus comportamentos de poupança, eles se diferenciam significativamente na atitude de fazer um empréstimo para comprar itens não necessários (Watson, 2003).

Rindfleisch e Burroughs (2004) sugeriram que o consumo pode ser usado, por exemplo, no desenvolvimento da identidade individual e na fixação dessa identidade, no contexto de experiências coletivas. Rose e Dejesus (2007) afirmam que o consumo pode ser usado como um meio para aumentar o sentimento de "pertencer", e aqueles que possuem essa necessidade podem ter predisposição para o materialismo.

A associação entre materialismo e o corpo foi abordada por Bartky (1982) em sua pesquisa sobre a experiência corporal das mulheres. O autor afirma que o complexo de moda/beleza está enraizado no capitalismo. Ao promover uma visão do corpo feminino como um objeto ou um projeto, fomenta-se uma cultura de consumo em que o próprio corpo, somado aos produtos e serviços que podem aumentar o seu valor, torna-se uma commodity. Os resultados de HendersonKing e Henderson-King (2005) também são condizentes com a análise de Bartky. As mulheres motivadas por aspirações materialistas, ao invés de aspirações intrínsecas, foram mais propensas a experimentar o corpo como um projeto, indicando que são mais favoráveis a ver a cirurgia estética como uma forma útil para aumentar a atratividade. Assim, neste estudo, postula-se que:

H1: As mulheres mais materialistas têm maior probabilidade de se submeterem a procedimentos cirúrgicos estéticos do que as mulheres menos materialistas.

REMark - Revista Brasileira de Marketing, São Paulo, v. 12, n. 4, p. 44-63, out/dez. 2013. 


\section{PROCEDIMENTOS METODOLÓGICOS}

A população desse estudo pode ser definida como mulheres das classes socioeconômicas A e B moradoras das cidades de São Paulo ou Recife. Essas cidades possuem características demográficas e econômicas distintas. Primeiramente, a cidade de São Paulo, também conhecida por “terra da garoa", está situada na região Sudeste do Brasil, pertence ao Estado de São Paulo e é considerada como o principal motor econômico-financeiro e centro de decisões corporativas da América Latina (Pimenta, 2007). Já a cidade de Recife, capital do estado de Pernambuco, é uma cidade litorânea e está situada na região Nordeste do país. A cidade atrai turistas do mundo todo por sua beleza natural e também por suas festas, de Carnaval e São João.

A amostra obtida é não probabilística: um link foi enviado pela rede social Facebook entre janeiro e fevereiro de 2012 para que moradoras dessas cidades pudessem preencher o questionário. A amostra obtida foi de 425 respondentes, mas 78 questionários foram descartados por estarem inválidos (por exemplo, a respondente não reside em São Paulo ou em Recife) ou incompletos. Assim, o total de respostas consideradas válidas para o estudo é de 347.

\subsection{A ESCALA DE AUTOESTIMA}

A escala de autoestima de Rosenberg (1965) foi traduzida e validada para o contexto brasileiro por Dini, Ferreira e Quaresma (2004) e possui dez itens (AE2 a AE11, apresentados no Figura 1 a seguir). Esses itens, além do AE1 ("Eu estou satisfeita com a minha aparência”), foram submetidos à análise fatorial exploratória para que sua estrutura dimensional fosse investigada.

\begin{tabular}{|c|l|}
\hline CÓDIGO & \multicolumn{1}{|c|}{ REDAÇÃO DO ITEM } \\
\hline AE1 & Eu estou satisfeita com a minha aparência. \\
\hline AE2 & De forma geral (apesar de tudo), estou satisfeita comigo mesma. \\
\hline AE3 & Às vezes, eu acho que eu não sirvo para nada (desqualificada ou inferior em relação aos outros). \\
\hline AE4 & Eu sinto que eu tenho um tanto (um nú mero) de boas qualidades. \\
\hline AE5 & Eu sou capaz de fazer coisas tão bem quanto a maioria das outras pessoas (desde que me ensinadas). \\
\hline AE6 & Não sinto satisfação nas coisas que realizei. Eu sinto que não tenho muito do que me orgulhar. \\
\hline AE7 & Às vezes, eu realmente me sinto inútil (incapaz de fazer as coisas). \\
\hline AE8 & Eu sinto que sou uma pessoa de valor, pelo menos num plano igual (nu m mes mo nível) às outras pessoas. \\
\hline AE9 & Não me dou o devido valor. Gostaria de ter mais respeito por mim mes ma. \\
\hline AE10 & Quase sempre estou inclinada a achar que sou uma fracas sada. \\
\hline AE11 & Eu tenho uma atitude positiva (pensamentos, atos e sentimentos positivos) em relação a mim mesma. \\
\hline
\end{tabular}

Figura 1 - Escala de Autoestima de Dini, Ferreira e Quaresma (2004) e itens submetidos à análise fatorial exploratória

Fonte: os autores, a partir de Dini, Ferreira, e Quaresma (2004).

REMark - Revista Brasileira de Marketing, São Paulo, v. 12, n. 4, p. 44-63, out/dez. 2013. 
A análise fatorial exploratória foi conduzida com extração de fatores pela técnica de componentes principais. Na solução inicial, três fatores apresentaram autovalores maiores que 1,00, daí a decisão por manter a solução final com três fatores. A saída abaixo se refere à solução final, rotacionada pela técnica VARIMAX.

Tabela 1 - Resultado da análise fatorial exploratória dos 11 itens de autoestima

\begin{tabular}{|c|c|c|c|c|}
\hline VARIÁVEL & FATOR1 & FATOR2 & FATOR3 & COMUNALIDADE \\
\hline AE7 & $-0,754$ & 0,021 & 0,043 & 0,571 \\
\hline AE3 & $-0,754$ & 0,094 & 0,115 & 0,59 \\
\hline AE10 & $-0,731$ & 0,187 & 0,01 & 0,57 \\
\hline AE9 & $-0,612$ & 0,048 & 0,251 & 0,44 \\
\hline AE6 & $-0,594$ & 0,116 & $-0,042$ & 0,368 \\
\hline AE1 & 0,535 & $-0,308$ & $-0,266$ & 0,452 \\
\hline AE5 & 0,053 & $-0,795$ & $-0,05$ & 0,638 \\
\hline AE8 & 0,125 & $-0,721$ & $-0,016$ & 0,536 \\
\hline AE4 & 0,184 & $-0,685$ & $-0,136$ & 0,521 \\
\hline AE1 & 0,094 & $-0,038$ & $-0,866$ & 0,754 \\
\hline AE2 & 0,114 & $-0,136$ & $-0,85$ & 6,1996 \\
\hline Variância & & & & 0,564 \\
\hline \% Var & 0,251 & 0,163 & 0,149 & \\
\hline
\end{tabular}

Fonte: elaborado pelos autores com auxílio do pacote estatístico Minitab 16.

Três itens, por possuírem comunalidades baixas, foram excluídos da análise (AE6, AE9 e AE11) e o processo descrito foi repetido. A saída do modelo reformulado é apresentada abaixo.

Tabela 2 - Resultado da análise fatorial exploratória de 8 itens da escala de autoestima

\begin{tabular}{|c|c|c|c|c|}
\hline VARIÁVEL & FATOR1 & FATOR2 & FATOR3 & COMUNALIDADE \\
\hline AE3 & $-0,824$ & 0,105 & $-0,135$ & 0,708 \\
\hline AE7 & $-0,822$ & 0,03 & $-0,089$ & 0,684 \\
\hline AE10 & $-0,723$ & 0,223 & $-0,005$ & 0,572 \\
\hline AE5 & 0,042 & $-0,789$ & 0,054 & 0,627 \\
\hline AE8 & 0,127 & $-0,731$ & 0,017 & 0,551 \\
\hline AE4 & 0,155 & $-0,697$ & 0,146 & 0,531 \\
\hline AE1 & 0,089 & $-0,037$ & 0,881 & 0,786 \\
\hline AE2 & 0,092 & $-0,147$ & 0,858 & 0,766 \\
\hline & & & & 5,2248 \\
\hline Variância & 1,9347 & 1,7271 & 1,5631 & 0,653 \\
\hline \% Var & 0,242 & 0,216 & 0,195 & \\
\hline
\end{tabular}

Fonte: elaborada pelos autores com auxílio do pacote estatístico Minitab 16.

REMark - Revista Brasileira de Marketing, São Paulo, v. 12, n. 4, p. 44-63, out/dez. 2013. 
O modelo com três fatores explica $65,3 \%$ da variabilidade dos dados e nenhum dos itens, individualmente, apresenta comunalidade abaixo de 0,500 .

O fator 1 está mais correlacionado com os itens AE3, AE7 e AE10. Pode ser batizado de "valorização do eu" (já que as cargas fatoriais de AE3, AE7 e AE10 com o fator 1 são negativas). O fator 2 está mais correlacionado com os itens AE4, AE5 e AE8. Pode ser batizado de "comparação social" (cabe lembrar que as cargas fatoriais de AE4, AE5 e AE8 com o fator 2 são negativas). Por fim, o fator 3 está mais correlacionado com os itens AE1 e AE2. Pode ser batizado de "satisfação com a aparência".

Em relação à confiabilidade interna, os valores do alfa de Cronbach, calculados considerando os itens destacados em cada fator, foram: 0,73 para "valorização do eu", 0,61 para "comparação social" e 0,71 para "satisfação com a aparência". O descarte de itens não elevou nenhum desses três valores, que podem ser considerados moderadamente satisfatórios, todos maiores que 0,60 , critério comumente estabelecido como mínimo para que haja evidência de que um construto seja unidimensional (Churchill, 1979; Pedhazur \& Schmelkin, 1991; Mazzocchi, 2008).

A partir do modelo apresentado na Tabela 2, foram geradas, no banco de dados disponível para análise, três novas variáveis, que representam os escores fatoriais dos participantes da pesquisa em cada um dos três fatores descritos ("valorização do eu", "comparação social" e "satisfação com a aparência"). Esses escores fatoriais foram utilizados nas análises apresentadas na seção Resultados.

\subsection{A ESCALA DE MATERIALISMO}

A escala utilizada para medir o materialismo dos respondentes neste trabalho foi a de Richins (2004). Adotou-se sua versão reduzida (de nove itens), a qual foi traduzida, adaptada e validada para o contexto brasileiro (em particular para o contexto paulistano) por Ponchio e Aranha (2008). Os itens da escala utilizada na pesquisa de campo, medidos na escala Likert de 5 pontos, são apresentados na Figura 2.

\begin{tabular}{|c|l|}
\hline CÓDIGO & \multicolumn{1}{|c|}{ REDAÇÃO DO ITEM } \\
\hline MAT1 & Eu ad miro pessoas que pos suem casa, carros e roupas caras. \\
\hline MAT2 & Eu gosto de gastar dinheiro com coisas caras. \\
\hline MAT3 & Minha vida seria melhor se eu tivesse coisas que eu não tenho. \\
\hline MAT4 & Co mprar coisas me dá mu ito prazer. \\
\hline MAT5 & Eu ficaria muito mais fe liz se eu pudesse comprar mais coisas. \\
\hline MAT6 & Eu gosto de possuir coisas que impres sionam as pessoas. \\
\hline MAT7 & Eu gosto de muito lu xo na minha vida. \\
\hline MAT8 & Me incomoda quando não posso comprar tudo que quero. \\
\hline MAT9 & Gastar muito dinheiro está entre as coisas mais importantes da vida. \\
\hline
\end{tabular}

Figura 2 - Escala de Materialismo de Richins (2004), adaptada por Ponchio e Aranha (2008)

Fonte: adaptado de Ponchio e Aranha (2008).

REMark - Revista Brasileira de Marketing, São Paulo, v. 12, n. 4, p. 44-63, out/dez. 2013. 
Resultados da análise fatorial exploratória conduzida com os itens dessa escala (com extração de fatores pela técnica de componentes principais e rotação VARIMAX) são apresentados na Tabela 3. Houve apenas um fator com autovalor maior que 1,00, critério de corte adotado para definir o número de fatores da solução apresentada.

Tabela 3 - Resultado da análise fatorial exploratória dos 9 itens da escala de materialismo

\begin{tabular}{|c|c|c|}
\hline VARIÁVEL & FATOR1 & COMUNALIDADE \\
\hline MAT1 & 0,617 & 0,381 \\
\hline MAT2 & 0,657 & 0,432 \\
\hline MAT3 & 0,682 & 0,465 \\
\hline MAT4 & 0,63 & 0,397 \\
\hline MAT5 & 0,724 & 0,524 \\
\hline MAT6 & 0,74 & 0,547 \\
\hline MAT7 & 0,76 & 0,578 \\
\hline MAT8 & 0,661 & 0,437 \\
\hline MAT9 & 0,593 & 0,352 \\
\hline Variância & & \\
\hline \%ar & 4,113 & \\
\hline
\end{tabular}

Fonte: elaborado pelos autores com auxílio do pacote estatístico Minitab 16.

Quatro itens, por possuírem comunalidades baixas, foram excluídos da análise (MAT1, MAT2, MAT4 e MAT9) e o processo descrito foi repetido. A saída do modelo reformulado é apresentada abaixo.

Tabela 4 - Resultado da análise fatorial exploratória de 5 itens da escala de materialismo

\begin{tabular}{|c|c|c|}
\hline VARIÁVEL & FATOR1 & COMUNALIDADE \\
\hline MAT3 & 0,742 & 0,55 \\
\hline MAT5 & 0,784 & 0,615 \\
\hline MAT6 & 0,748 & 0,56 \\
\hline MAT7 & 0,732 & 0,536 \\
\hline MAT8 & 0,74 & 0,547 \\
\hline Variância & \\
\hline \% Var & \multicolumn{2}{|c|}{2,8077} \\
\hline
\end{tabular}

Fonte: elaborado pelos autores com auxílio do pacote estatístico Minitab 16.

O valor do alfa de Cronbach para a escala (com cinco itens) é de 0,8043. A partir do modelo apresentado na Tabela 4, foi gerada, no banco de dados disponível para análise, nova variável, que representa o escore fatorial dos participantes da pesquisa na escala de materialismo. Esses escores fatoriais foram utilizados nas análises apresentadas na seção Resultados.

REMark - Revista Brasileira de Marketing, São Paulo, v. 12, n. 4, p. 44-63, out/dez. 2013. 


\section{RESULTADOS}

Das 347 respondentes, 173 são da cidade de São Paulo e 174, da cidade de Recife. A distribuição da variável idade é apresentada na Figura 3 a seguir.

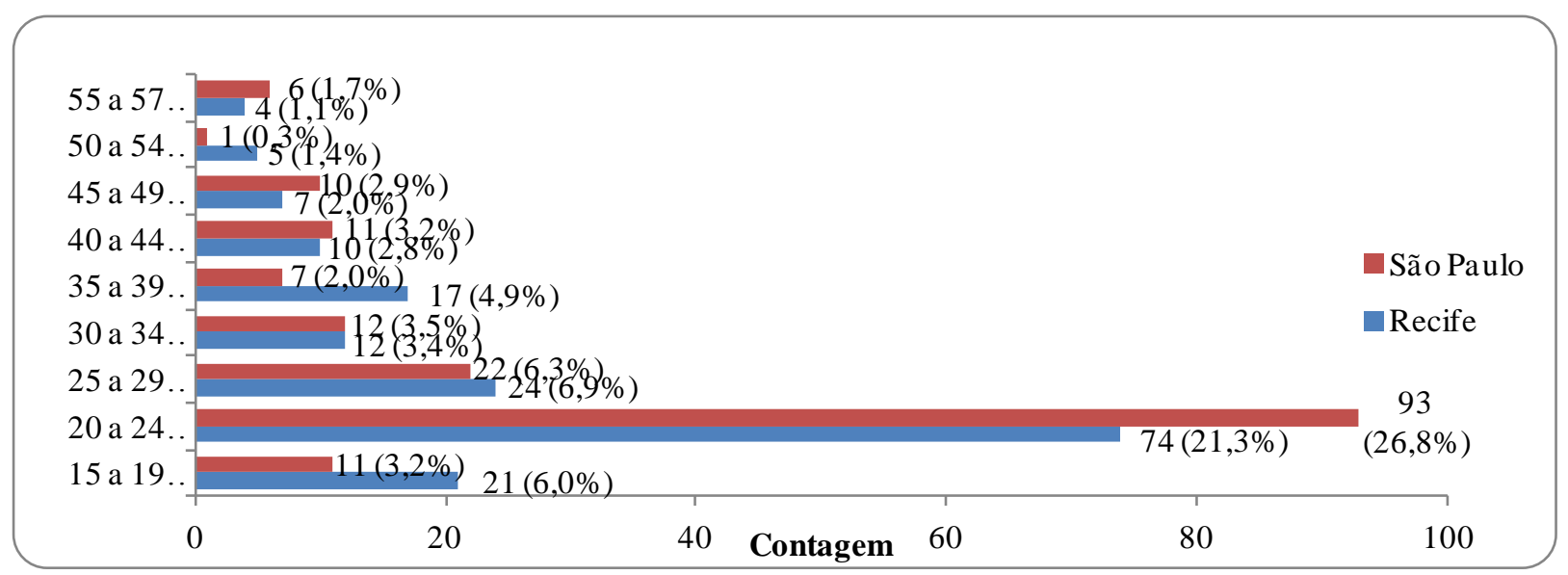

Figura 3 - Faixa Etária das Respondentes

Fonte: Elaboração própria.

Assim, a faixa etária de 20 a 24 anos prevaleceu entre nossas respondentes, o que representou $48,1 \%$ (167 mulheres) do total.

Há na amostra 59 mulheres (17\% das respondentes) que já fizeram algum procedimento cirúrgico estético; destas mulheres destaca-se que 56\% (33 mulheres) eram de Recife e 44\% (26 mulheres) de São Paulo.

Das cirurgias que essas mulheres realizaram, $46 \%$ (42 mulheres) foram nos seios, $14 \%$ (13 mulheres) no abdômen, $11 \%$ no nariz (10 mulheres) e 9\% nas pernas (oito mulheres). Dentre as que fizeram cirurgia nos seios, $68 \%$ (28 mulheres) colocaram implantes de silicone.

A Tabela 5 a seguir apresenta a matriz de correlação entre as variáveis quantitativas do estudo. Para ilustrar a associação entre as variáveis quantitativas e qualitativas, são apresentados dois box-plots na Figura 4.

Tabela 5 - Coeficientes de correlação entre as variáveis do estudo

\begin{tabular}{|c|c|c|c|c|}
\hline & AE-FATOR1 & AE-FATOR2 & AE-FATOR3 & MATERIALIS MO \\
\hline Idade & $0,125^{*}$ & $-0,029(\mathrm{~ns})$ & $0,071(\mathrm{~ns})$ & $0,051(\mathrm{~ns})$ \\
\hline AE-fator1 & - & $0,000(\mathrm{~ns})$ & $0,000(\mathrm{~ns})$ & $-0,141^{* *}$ \\
\hline AE-fator2 & & - & $0,000(\mathrm{~ns})$ & $0,051(\mathrm{~ns})$ \\
\hline AE-fator3 & & & - & $-0,218^{* *}$ \\
\hline Materialismo & & & & - \\
\hline
\end{tabular}

*Significante ao nível de 0,05 ;**Significante ao nível de 0,01 ; (ns) não significante.

Fonte: elaboração própria.

REMark - Revista Brasileira de Marketing, São Paulo, v. 12, n. 4, p. 44-63, out/dez. 2013. 
Percebe-se que os coeficientes de correlação linear entre materialismo e as dimensões da escala de autoestima são baixos, o que reforça a ideia de validade discriminante das escalas empregadas no estudo. Por construção (em decorrência de os fatores serem ortogonais), as correlações entre as dimensões de autoestima são nulas.

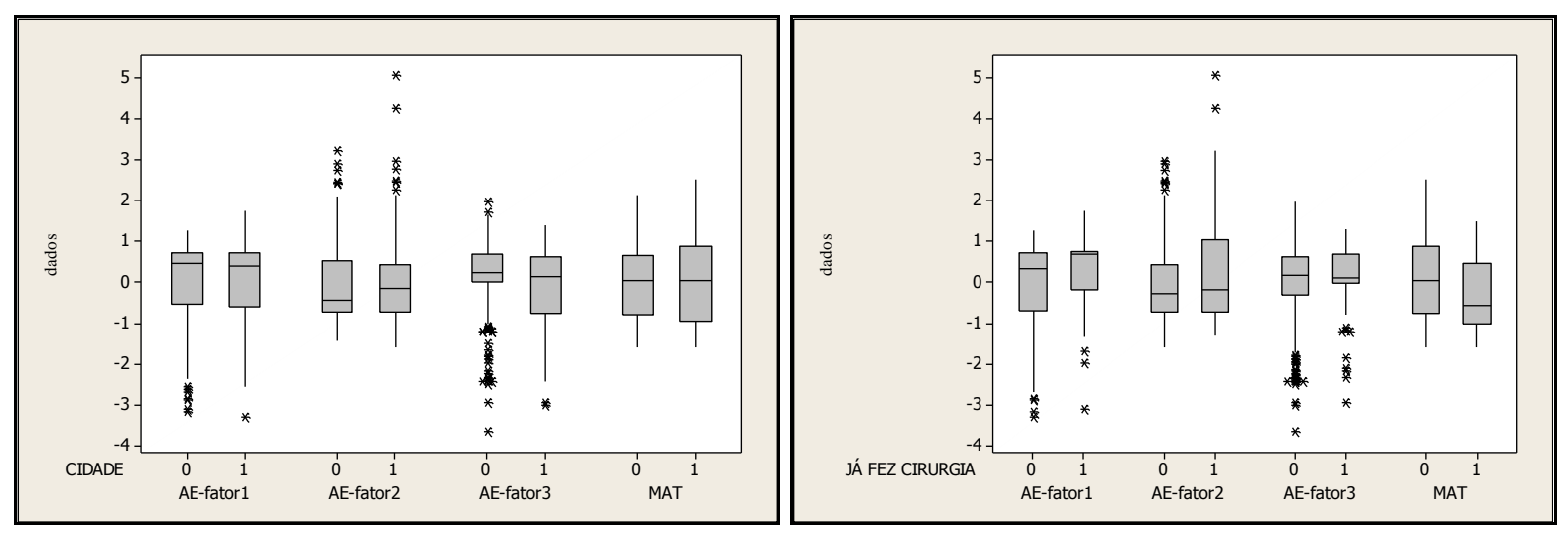

Cidade: 0 = Recife; 1 = São Paulo. 'Já fe z cirurg ia': 0 = não; $1=\operatorname{sim}$.

Figura 4 - Box-plots para ilustrar as distribuições das variáveis quantitativas do estudo por nível das variáveis 'cidade' e 'já fez cirurgia'

Fonte: elaboração própria com auxílio do pacote estatístico Minitab 16.

\subsection{MODELO DE REGRESSÃo LOGÍSTICA BINÁRIA PARA PREVER REALIZAÇÃo DE CIRURGIA PLÁSTICA ESTÉTICA}

Com o intuito de compreender quais variáveis possuem as sociação com o ato de realizar cirurgia plástica, ajustou-se aos dados de estudo um modelo de regressão logística binária, cuja variável resposta, qualitativa, tem duas possibilidades de ocorrência: a respondente já realizou ou nunca realizou uma cirurgia estética. Esses casos foram codificados com 1 e 0 , respectivamente. As variáveis independentes foram cidade da respondente $(0=$ Recife e 1 = São Paulo), AE-fator1 ("valorização do eu"), AE- fator2 ("comparação social"), AE- fator3 ("satisfação com a aparência") e materialismo. A Tabela 6 sintetiza as principais informações do modelo final:

REMark - Revista Brasileira de Marketing, São Paulo, v. 12, n. 4, p. 44-63, out/dez. 2013. 
Tabela 6 - Regressão logística binária: realização de cirurgia plástica

\begin{tabular}{|c|c|c|c|c|c|}
\hline \multicolumn{3}{|c|}{ Variável resposta } & Valor & \multicolumn{2}{|l|}{ Contagem (*) } \\
\hline \multirow{2}{*}{\multicolumn{3}{|c|}{ Você já realizou cirurgia plástica? }} & 1 & 59 & \multirow[t]{3}{*}{ (Evento) } \\
\hline & & & 0 & 288 & \\
\hline & & & Total & 347 & \\
\hline Preditor & Coeficiente & $p$ & Razão de Chance & I.C. $95 \%$ inf. & I.C. $95 \%$ sup. \\
\hline Constante & $-1,5791$ & & & & \\
\hline Cidade & $-0,3168$ & 0,288 & 0,73 & 0,41 & 1,31 \\
\hline AE-fator1 & 0,3078 & 0,066 & 1,36 & 0,98 & 1,89 \\
\hline AE-fator2 & 0,3389 & 0,012 & 1,40 & 1,08 & 1,83 \\
\hline AE-fator3 & $-0,0872$ & 0,573 & 0,92 & 0,68 & 1,24 \\
\hline Materialismo & $-0,4945$ & 0,003 & 0,61 & 0,44 & 0,84 \\
\hline
\end{tabular}

Fonte: elaborada pelos autores com auxílio do pacote estatístico Minitab 16.

A equação de regressão encontrada foi a seguinte:

Ou, expressa de outra maneira,

onde: $\boldsymbol{p}_{\boldsymbol{i}}=$ probabilidade de realização de cirurgia plástica pela respondente ${ }_{i}$ cidade $_{\mathrm{i}}, \mathrm{AE}$-fator $1_{\mathrm{i}}, \mathrm{AE}$-fator $2_{\mathrm{i}}, \mathrm{AE}$ - fator $3_{\mathrm{i}}$ e materialis $\mathrm{mo}_{\mathrm{i}}=$ valor destas variáveis medidas para a respondente ${ }_{i}$.

A estatística G, que testa se todos os coeficientes do modelo de regressão são iguais a zero, foi de 20,91, significante ao nível de 0,001. Logo, há evidência de que pelo menos um dos coeficientes do modelo de regressão é diferente de zero. Observando-se os p-valores dos coeficientes das variáveis, constata-se haver evidência de que os associados às variáveis AE-fator2 e materialismo são diferentes de zero, ao nível de $5 \%$.

Os testes de goodness-of-fit de Pearson e de Hosmer-Lemeshow tiveram p-valores de 0,383 e de 0,623 , respectivamente, indicando que não há evidência para afirmar que o modelo não se ajusta aos dados adequadamente. Quanto às medidas de associação, o percentual de pares concordantes foi de $66,3 \%$, resultando em um coeficiente de Somers' D igual a 0,33.

De acordo com o modelo, quanto mais materialista a respondente, menor a probabilidade de ter realizado cirurgia plástica. De acordo com a literatura, espera-se que mulheres mais materialistas

REMark - Revista Brasileira de Marketing, São Paulo, v. 12, n. 4, p. 44-63, out/dez. 2013. 
tenham maior probabilidade de realizar cirurgia estética. Este resultado dissonante, em direção contrária à hipótese H1 formulada, incita novas investigações. Talvez, a escala de materialismo, desenvolvida para o contexto da população paulistana de baixa renda (Ponchio \& Aranha, 2008), não tenha tido bom desempenho para mensurar o construto entre mulheres paulistanas e recifenses das classes socioeconômicas A e B (quatro dos nove itens da escala foram descartados neste estudo). Além disso, pode-se especular a existência do efeito das respostas socialmente desejáveis, relatado por Mick (1996) e associado à aplicação da escala de materialismo: as pessoas atribuem respostas aos itens de materialismo de acordo com como gostariam de ser vistas, e não necessariamente de acordo com seus valores e atitudes.

Parece uma contribuição substantiva que a variável 'AE-fator2' (batizada de “comparação social") tenha permanecido no modelo. Quanto maior a pontuação da respondente nessa variável, maior a probabilidade de que tenha realizado cirurgia plástica. $\mathrm{O}$ fato de as demais dimensões da escala de autoestima não terem sido significantes neste modelo sugere que a dimensão social se sobreponha à dimensão pessoal na decisão por efetuar cirurgias plásticas.

\section{CONSIDERAÇÕES FINAIS}

A partir dos resultados apresentados, nota-se que a probabilidade de ter realizado cirurgia estética é parcialmente explicada por alguma preocupação social, em linha com os estudos de Vanzellotti (2007) e Teixeira (2001).

Segundo Del Priore (2000), na tentativa de possuir melhor imagem de si a mulher busca remodelar seu corpo. A busca incessante da melhor imagem feminina é uma forma de aceitação, uma vez que a opinião alheia costuma influenciar a autoestima. As modificações para se atingir um corpo ideal tendem a aumentar a autoestima feminina visto que a partir desses recursos a distância entre o Eu real e o Eu ideal será diminuída (Xue, Zhou, \& Zhou, 2002).

Pode ser caracterizado como limitação do estudo o fato de este ser transversal e não longitudinal. Deve-se atentar, ainda, para o desempenho inferior ao desejado da escala de materialismo, bem como para a possível influência de respostas socialmente desejáveis quando se mensura este construto (Mick, 1996; Ponchio \& Aranha, 2008). A associação verificada entre materialismo e a probabilidade de ter realizado cirurgia estética, contrária à esperada, merece futuras investigações. Talvez, a mensuração da intenção de realizar procedimentos cirúrgicos estéticos seja uma estratégia mais adequada a estudos de corte trans versal do que apenas verificar se

REMark - Revista Brasileira de Marketing, São Paulo, v. 12, n. 4, p. 44-63, out/dez. 2013. 
a respondente já realizou ou não cirurgia estética, dado que os valores e as atitudes dos consumidores mudam ao longo de suas vidas.

Como sugestão para próximas investigações, seria interessante a realização de um estudo longitudinal para acompanhar a atual geração de meninas jovens, visando verificar qual proporção delas fará cirurgia plástica, bem como a evolução da autoestima e do materialismo ao longo das fases de suas vidas. 


\section{REFERÊNCIAS}

Arnould, E. J., \& Thompson, C. J. (2005). Consumer Culture Theory (CCT): Twenty Years of Research. Journal of Consumer Research, 31(4), 868-882.

ASAPS - American Society of Aesthetic Plastic Surgery. (2010). Demand for Plastic Surgery Rebounds by Almost 9\%. Acesso 12 abr. 2012, em http://www.cosmeticplasticsurgerystatistics.com/statistics.html\#2010-HIGHLIGHTS .

Bartky, S. L. (1982). Narcissism, femininity, alienation. Social Theory and Practice, 8(2), 127-144.

Belk, R. W. (1983). Worldly Possessions: Issues and Criticisms. Advances in Consumer Research, 10(1), 514-519.

Belk, R. W. (1984). Three Scales to Measure Constructs Related to Materialism: Reliability, Validity, and Relationships to Measures of Happiness. Advances in Consumer Research, 11(1), 291-297.

Belk, R. W. (1988). Possessions and the Extended Self. Journal of Consumer Research, 15(2), 139168.

Birdwell, A. E. (1968). A study of the influence of image congruence on consumer choice. The Journal of Business, 41(1), 76-88.

Brasil Ocupa $2^{\mathrm{a}}$ Posição em número de cirurgias plásticas estéticas no mundo. (2013, janeiro 24). Veja, disponível em http://veja.abril.com.br/noticia/saude/brasil-ocupa-2a-posicao-em-numerode-cirurgias-plasticas-esteticas-no- mundo .

Brown, A., Furnham, A., Glanville, L., \& Swami, V. (2007). Factors that affect the likelihood of undergoing cosmetic surgery. Aesthetic Surgery Journal, 27(5), 501-508.

Burgess, S., \& Steenkamp, J. B. E. (2006). Marketing renaissance: how research in emerging markets advances marketing science and practice. International Journal of Research in Marketing, 23(4), 337-356.

Churchill, G. A. (1979). Paradigm for Developing Better Measures of Marketing Constructs. Journal of Marketing Research, 16(1), 64-73.

Crockett, R. J., Pruzinsky, T., \& Persing, J. A. (2007). The influence of plastic surgery 'reality TV' on cosmetic surgery patient expectations and decision making. Plastic and Reconstructive Surgery, 120(1), 316-324.

Del Priore, M. (2000). Corpo a corpo com a mulher: pequena história das transformações do corpo feminino no Brasil. São Paulo, Brasil: SENAC.

REMark - Revista Brasileira de Marketing, São Paulo, v. 12, n. 4, p. 44-63, out/dez. 2013. 
Delinsky, S. (2005). Cosmetic Surgery: A Common and Accepted Form of Self-lmprovement. Journal of Applied Social Psychology, 35(10), 2012-2028.

Dini, G. M., Quaresma, M. R., \& Ferreira, L. M. (2004). Adaptação cultural e Validação da Versão Brasileira da Escala da Auto-Estima de Rosenberg. Revista da Sociedade Brasileira de cirurgia plástica, 19(1), 41-52.

Euromonitor. (2010). Consumer Lifestyles - Brazil. Acesso 10 dez. 2011, em http://www.portal.euromonitor.com/Portal/Pages/Search/SearchResultsList.aspx .

Fournier, S., \& Richins, M. L. (1991). Some Theoretical and Popular Notions Concerning Materialism. Journal of Social Behavior and Personality, 6(6), 403-414.

Heath, A. P., \& Scott, D. (1997). The self-concept and image congruence hypothesis - An empirical evaluation in the motor vehicle market. European Journal of Marketing, 32(10), 1110-1123.

Henderson-King, D., \& Brooks, K. (2009). Materialism, sociocultural appearance messages, and parental attitudes predict college women's attitudes about cosmetic surgery. Psychology of Women Quarterly, 33(1), 133-142.

Henderson-King, D., \& Henderson-King, E. (2005). Acceptance of cosmetic surgery: Scale development and validation. Body Image, 2(2), 137-149.

Hirschman, E., \& Holbrook, M. B. (1981). Symbolic Consumer Behavior: an introduction. Symbolic Consumer Behavior, Association for Consumer Research, New York, NY, 1-2.

Holbrook, M. B., \& Hirschman, E. C. (1982). The Experiential Aspects of Consumption: Consumer Fantasies, Feelings, and Fun. Journal of Consumer Research, 9(2), 132-140.

Machado, M. V., \& Pereira, S. N. J. (2010). Espelho, Espelho meu, Quem Sou Eu? Consumo Estético e a Construção da Identidade da Mulher. Anais eletrônicos do IV Encontro de Marketing da ANPAD [CD-ROM], Florianópolis, Brasil, 4, 1-16.

Mazzocchi, M. (2008). Statistics for Marketing and Consumer Research. Thousand Oaks, CA: SAGE Publications.

McCracken, G. (2003). Cultura e Consumo: novas abordagens ao caráter simbólico dos bens e das atividades de consumo. Rio de Janeiro, Brasil: Mauad.

Mick, D. G. (1996). Are Studies of Dark Side Variables Confounded by Socially Desirable Responding? The Case of Materialism. Journal of Consumer Research, 23(2), 106-119.

Mittal, B. (2006). I, me, and mine - how products become consumers' extended selves. Journal of Consumer Behaviour, 5(6), 550-562.

Pedhazur, E., \& Schmelkin, L. (1991). Measurement, Design, and Analysis: an integrated approach. Hillsdale, New Jersey: Lawrence Erlbaum Associates.

REMark - Revista Brasileira de Marketing, São Paulo, v. 12, n. 4, p. 44-63, out/dez. 2013. 
Pimenta, A. (2007). Esqueça os países. O poder está com as cidades. Revista Exame. Acesso 3 out. 2011, em http://exame.abril.com.br/revista-exame/edicoes/0907/noticias/esqueca-os-paises-opoder-esta-com-as-cidades-m0144514 .

Ponchio, M. C., \& Aranha, F. (2008). Materialism as a predictor variable of low income consumer behavior when entering into installment plan agreements. Journal of Consumer Behaviour, $7(1), 21-34$.

Richins, M. L. (2004). The material values scale: Measurement properties and development of a short form. Journal of Consumer Research, 31(1), 209-219.

Richins, M. L., \& Dawson, S. (1992). A Consumer Values Orientation for Materialism and Its Measurement: Scale Development and Validation. Journal of Consumer Research, 19(3), 303316.

Rindfleisch, A., \& Burroughs, J. E. (2004). Terrifying thoughts, terrible materialism? Contemplations on a terror management account of materialism and consumer behavior. Journal of Consumer Psychology, 14(3), 219-224.

Rose, P., \& Dejesus, S. P. (2007). A model of motivated cognition to account for the link between self-monitoring and materialism. Psychology \& Marketing, 24(2), 93-115.

Rosenberg, M. (1965). Society and the adolescent self-image. Princeton: Princeton University Press.

Rosenberg, M., \& Pearlin, L. (1978). Social Class and Self-Esteem among Children and Adults. American Journal of Sociology, 84(1), 53-77.

Rosenberg, M., Schooler, C., \& Schoenbach, C. (1989). Self-Esteem and Adolescent Problems: Modeling Reciprocal Effects. American Sociological Association, 54(6), 1004-1018.

Sarwer, D. B., Larossa, D., Bartlett, S. P., Low, D. W., Bucky, L. P., \& Whitaker, L. A. (2003). Body image concerns of breast augmentation. Plastic Reconstructive Surgery, 112(1), 83-90.

Slater, D. (2002). Cultura de consumo e modernidade. São Paulo, Brasil: Nobel.

Solomon, M. R. (2008). O Comportamento do Consumidor: comprando, possuindo e sendo. São Paulo, Brasil: Bookman.

Steenkamp, J. B. E. (2005). Moving out of the US Silo: a call to arms for conducting international marketing research. Journal of Marketing, 69(4), 6-8.

Swami, V., Campana, A., \& Coles, R. (2012). Acceptance of cosmetic surgery among British female university students: are there ethnic differences? European Psycholog ist, 17(1), 55-62.

REMark - Revista Brasileira de Marketing, São Paulo, v. 12, n. 4, p. 44-63, out/dez. 2013. 
Swami, V., Chamorro-Premuzic, T., Bridges, S., \& Furnham, A. (2009). Acceptance of cosmetic surgery: Personality and individual difference predictors. Body Image, 6(1), 7-13.

Teixeira, S. A. (2001). Produção e consumo social da beleza. Horizontes Antropológicos, 7(16), 189-220.

Vanzellotti, C. A. (2007). Esperança para dar e vender: a esperança no consumo de cosméticos antissinais. Dissertação de Mestrado, Universidade Federal do Rio Grande do Sul, Porto Alegre, Brasil.

Watson, J. (2003). The Relationship of Materialism to Spending Tendencies, Saving and Debt. Journal of Economic Psychology, 24(6), 723-739.

Wattanasuwan, K. (2005). The Self and Symbolic Consumption. Journal of American Academy of Business, 6(1), 179-184.

Xue, F., Zhou, S., \& Zhou, P. (2002). Self-esteem, life-satisfaction and materialism: Effects of advertising images on Chinese college students. In C. R. Taylor (Ed.), New Directions in International Advertising Research - Advances in International Marketing, Volume 12 (pp. 243-261). Bingley, UK: Emerald Group Publishing Limited.

Data do recebimento do artigo: 24/06/2013

Data do aceite de publicação: 02/10/2013

REMark - Revista Brasileira de Marketing, São Paulo, v. 12, n. 4, p. 44-63, out/dez. 2013. 